\title{
I Plan Integral para el Fomento de la Artesanía en Andalucía
}

Con el objetivo de ordenar y promocionar la actividad económica del sector artesano en la Comunidad Autónoma de Andalucía, la Consejería de Turismo, Comercio y Deporte está desarrollando la Ley de Artesanía de Andalucía, aprobada a finales de 2005. Entre otras actuaciones, ha elaborado, en colaboración con el equipo de investigadoras del Instituto de Desarrollo Regional, Fundación Universitaria, el I Plan Integral para el Fomento de la Artesanía de Andalucía (el primero que ha visto la luz en el ámbito nacional).

La metodología utilizada para su elaboración, dada la escasez de fuentes estadísticas de información oficiales y las particularidades del sector, ha sido plural y participativa, y su aplicación ha dado como resultado un exhaustivo análisis de la situación del sector, permitiendo establecer un diagnóstico sobre sus necesidades y potencialidades en Andalucía.

En este marco, mediante el empleo simultáneo de distintos métodos y técnicas cuantitativos y cualitativos complementarios (encuestas, entrevistas en profundidad, mesas técnicas de trabajo y la consulta a expertos), se ha recopilado la información necesaria para el análisis de la importancia socioeconómica del sector productivo de la artesanía, en el ámbito de la economía regional, y un diagnóstico que identifica las dificultades con las que se enfrenta este sector.

En la elaboración del Plan se consultó a las organizaciones representativas del empresariado y de los trabajadores, movilizando a una parte muy importante de los artesanos y artesanas de toda la región (con más de 150 participantes en las mesas técnicas de trabajo, celebradas en cada una de las provincias andaluzas). Asimismo, para obtener el mayor número posible de opiniones sobre la situación del sector, se ha realizado una encuesta personal a miembros de 625 talleres artesanos.

Esta movilización ha supuesto un factor de comunicación y movilización de los agentes vinculados al Plan. La participación en este proceso ha servido, además, para que los artesanos y artesanas, así como los agentes socioeconómicos participantes adquieran mayor conciencia del contexto en el que se desarrolla su actividad y se vean estimulados a la participación desde el principio. La información generada por los participantes ha proporcionado elementos claves de valoración y decisión, y, en definitiva, ha posibilitado el acceso al discurso cualitativo, tan apreciado en las tareas de diagnóstico y planificación.

El Plan tiene como objetivo final el incremento de la capacidad competitiva de las empresas de artesanía de Andalucía, cuyo logro se apoya en la consecución de los siguientes objetivos intermedios: adecuar los recursos humanos, materiales y tecnológicos de las empresas de artesanía a los requerimientos del mercado; fomentar las relaciones de cooperación y el asociacionismo entre las empresas de artesanía; desarrollar la ordenación y fomento de la actividad artesana; incrementar la información e investigación del sector artesano.

La variedad de aportaciones realizadas desde el propio sector ha permitido consensuar una serie de medidas que se desarrollarán en el marco cronológico previsto por el Plan entre 2007 y 2010.

Asi pues, estamos ante un amplio proceso participativo que ha permitido sentar las bases para revitalizar una actividad tradicional, conservando sus señas de identidad, sin dejar de lado la modernización del sector. El Plan supone un instrumento que facilitará el trabajo artesano y aumentará su competitividad, apoyando la formación de los nuevos trabajadores, la innovación tecnológica y los mecanismos de cooperación. En definitiva, el Plan Integral para el Fomento de la Artesanía en Andalucía supone un esfuerzo común por el futuro de una actividad con historia.

Cristina Campayo Rodriguez, María José Guerrero Mayo, Purificación López Igual, María José Dorado Rubín Equipo de Investigación del Instituto de Desarrollo Regional, Fundación Universitaria 